\title{
Élaboration d'un procédé de fabrication industrielle de surfaces asphériques de grande précision en verre
}

\author{
Stéphane Di Luzio ${ }^{1}$, Philippe Revel ${ }^{2, a}$, Henri Gagnaire ${ }^{3}$ et Bernard Feraud $^{4}$ \\ 1 Cour des Miracles, 69510 Rontalon, France \\ 2 Laboratoire Roberval, Unité de Recherche en Mécanique, FRE UTC/CNRS 2833, Université de Technologie de Compiègne, \\ 60200 Compiègne, France \\ 3 Laboratoire du Traitement du Signal et Instrumentation, Université Jean Monnet, LTSI/Filière Vision, 10 rue Barrouin, \\ 42000 Saint-Étienne, France \\ 4 Thales Angénieux, 42570 St Héand, France
}

Reçu le 10 juin 2005, accepté le 19 février 2007

\begin{abstract}
Résumé - La société Thales Angénieux souhaite intégrer des dioptres asphériques dans les systèmes optiques complexes de grande qualité qu'elle fabrique. C'est à la fois un enjeu technologique et commercial. Ces dioptres asphériques sont soumis aux mêmes critères de forme et de rugosité que les dioptres sphériques ou plans. Cependant, ils ne peuvent être fabriqués avec les méthodes conventionnelles utilisées pour les autres dioptres. Il a donc été nécessaire d'utiliser des techniques de production différentes. Parmi les techniques envisageables, Thales Angénieux a choisi de développer un processus de fabrication industrielle articulé autour de deux procédés : la micro-rectification et la finition magnéto-rhéologique. Pour atteindre le niveau final de qualité requis, il s'est avéré indispensable d'intercaler une phase de transition de polissage souple entre la micro-rectification et la finition magnéto-rhéologique. Ce nouveau processus de fabrication permet d'une part de réaliser des dioptres asphériques dont la forme présente un $P R t$ de l'ordre de $0,150 \mu \mathrm{m}$ et pour la rugosité un $R a$ inférieur à $3 \mathrm{~nm}$, et s'avère d'autre part parfaitement utilisable en milieu industriel.
\end{abstract}

Mots clés : Asphérique / verre / micro-rectification / finition / polissage / magnéto-rhéologie / fabrication

Abstract - An industrial process of high precision aspherical lens surfaces manufacturing. Aspherical surfaces are more and more used in high precision optical systems of Thales Angénieux. They must satisfy the same quality standards of form and roughness as the other dioptre surfaces. However, aspherical dioptres can not be manufactured with conventional methods used for spherical or plane surfaces, because form and roughness required can not be obtained simultaneously. Among the various available techniques, Thales Angénieux has chosen to develop an industrial manufacturing process, with two successive methods: micro-grinding and magneto-rheological finishing. Furthermore, micro-grinding phase is divided into two parts: firstly, a brittle one with a wheel with large size abrasive grains, secondly a ductile one with a wheel with small size abrasive grains. However, a transition phase, flexible polishing, was inserted between micro-grinding and magneto-rheological finishing phases to reach the objectives. Finally, this manufacturing process made it possible to produce aspherical glass surfaces with a defect of form $(P V)$ around $0.150 \mu \mathrm{m}$ and a roughness $(R a)$ less than $3 \mathrm{~nm}$. Moreover, this process can be integrated in an industrial manufacturing process.

Key words: Aspherical / glass / micro-grinding finishing / polishing / magneto-rheological / manufacturing

\section{Nomenclature}

Les paramètres de forme sont notés PRt et Prms et les paramètres de rugosité $R t, r m s$ et $R a$. La définition de $R t$ et PRt ainsi que rms et Prms sont similaires, seules

a Auteur correspondant : philippe.revel Qutc.fr changent la dimension de la surface mesurée et la surface de référence.

- PRt ou Rt : écart entre le point le plus haut et le point le plus bas de la surface mesurée par rapport à la surface de référence (unité : le micromètre).

- Prms ou rms : écart quadratique moyen (unité : le micromètre). 
Pour une mesure surfacique : rms = $\sqrt{\frac{1}{S} \iint_{S} z^{2}(x, y) \mathrm{d} x \mathrm{~d} y}$

- Ra : moyenne arithmétique (unité : le nanomètre). Pour une mesure en deux dimensions, ce paramètre est calculé ainsi : $R a=\frac{1}{L} \int_{0}^{L}|z(x)| \mathrm{d} x$.

\section{Introduction}

Les travaux présentés dans cet article ont été réalisés au sein de la société Thales Angénieux. Cette entreprise est spécialisée dans la conception et la réalisation de systèmes optiques complexes et performants (objectifs de caméras professionnelles, jumelles, systèmes de visée).

Jusqu'à présent, les systèmes optiques de grande qualité fonctionnant dans le domaine du visible comportent essentiellement des lentilles à dioptres plans ou sphériques. L'introduction de dioptres asphériques dans ces combinaisons optiques permet d'améliorer les performances optiques et/ou de diminuer le poids et l'encombrement de ces systèmes. L'objectif poursuivi durant ces travaux était de fabriquer industriellement des lentilles en verre possédant un dioptre asphérique répondant à des critères très stricts de qualité sur leur forme et leur rugosité.

Dans une première partie, nous rappellerons plus précisément ce qu'est un dioptre asphérique, et quelles sont les principales techniques et machines qui permettent de les élaborer. Les matériaux utilisés ainsi que les différents mécanismes d'enlèvement de matière pour un matériau fragile comme le verre seront ensuite décrits. Enfin, les résultats obtenus permettant de valider le processus de fabrication seront présentés et discutés.

\section{2 Élaboration de dioptres asphériques}

\subsection{Cahier des charges des dioptres asphériques}

Les définitions d'une surface (ou dioptre) asphérique de révolution et de ses paramètres sont rappelées dans l'Annexe 1.

Les lentilles, possédant un dioptre asphérique, susceptibles d'être utilisées dans les zooms de caméras à usage professionnel de Thales Angénieux ont un diamètre qui peut varier de 20 à $200 \mathrm{~mm}$. Ces dioptres asphériques peuvent avoir une asphéricité comprise entre $60 \mu \mathrm{m}$ et $1 \mathrm{~mm}$ (cf. Annexe 1). Ils doivent vérifier des critères de qualité en forme et en rugosité, critères tout à fait semblables à ceux appliqués aux dioptres sphériques présents dans les mêmes systèmes optiques. Les dioptres asphériques doivent donc satisfaire les tolérances suivantes :

- Rugosité : $R a<5 \mathrm{~nm}$,

- Forme : PRt $\leq 0,3 \mu \mathrm{m}$, Prms $\leq 0,03 \mu \mathrm{m}$.

\subsection{Choix d'un processus de fabrication}

Différentes techniques de fabrication de dioptres asphériques existent. Les unes sont des procédés de tournage à l'outil diamant sur des machines de haute précision, procédés utilisables sur des matériaux tels que le germanium, la fluorine, le sulfure de zinc ou le séléniure de zinc, mais pas sur les verres optiques [1]. D'autres techniques comme l'usinage laser [2], l'usinage au jet d'eau [3] et l'érosion ionique [4], sont essentiellement des techniques de laboratoire et ne peuvent encore être incluses dans un processus industriel. Certaines techniques conventionnelles de fabrication des dioptres sphériques ont été adaptées à la production de dioptres asphériques, mais comme elles demandent un savoir-faire très pointu de la part de l'opérateur, elles donnent des résultats difficilement reproductibles $[5,6]$. Il est également possible de créer des dioptres asphériques par dépôt sous vide. Cette technique fonctionne bien sur certains matériaux comme le germanium et pour des dioptres ayant une faible asphéricité $(20 \mu \mathrm{m})$ et un grand diamètre de l'ordre de $100 \mathrm{~mm}$, mais cela ne répond pas au cahier des charges défini précédemment [7]. Enfin, le moulage (ou pressage à chaud) qui consiste à chauffer une ébauche puis à la déformer est un procédé très prometteur, en constante évolution; cependant, lors de la prise de décision des techniques de fabrication utilisées pour réaliser les dioptres asphériques, ce procédé présentait trop de limitations pour être retenu [8].

La société Thales Angénieux a choisi de développer un processus de fabrication s'articulant autour de deux techniques de fabrication :

- la micro-rectification [9],

- la finition magnéto-rhéologique [10-12].

Ce processus doit permettre de produire dans un contexte industriel des dioptres asphériques convexes en respectant les tolérances exigées.

Le principe de la magnéto-rhéologie est exposé dans l'Annexe 2.

\subsection{Description du processus de fabrication}

Dans un premier temps, la réalisation des dioptres asphériques en verre a été divisée en trois phases : deux phases de micro-rectification et une phase de finition magnéto-rhéologique.

\subsubsection{Différentes étapes du processus initial}

Avant d'entamer la première phase de ce processus, la pièce est ébauchée de façon conventionnelle, par meulage, au rayon de meilleure sphère de la surface asphérique (cf. Annexe 1). Les trois étapes ont ensuite chacune des objectifs propres :

- La première étape de micro-rectification permet de passer d'un dioptre sphérique à un dioptre 
asphérique. Le principal objectif de cette étape, dite d'asphérisation, est d'ôter le maximum de matière en un minimum de temps, au détriment de l'état de surface obtenu après cette opération.

- La deuxième étape de micro-rectification a un double objectif. Le principal est d'améliorer la rugosité du dioptre : $R a \leq 20 \mathrm{~nm}, R t<200 \mathrm{~nm}$; une telle rugosité autorise alors la réalisation de mesures optiques. Le second objectif est d'améliorer la forme du dioptre.

- Enfin, la troisième étape est la finition magnétorhéologique du dioptre. Cette phase doit permettre d'atteindre la forme et la rugosité finales du dioptre.

\subsubsection{Machines de fabrication}

Les machines utilisées durant les phases de microrectification et de magnéto-rhéologie sont des machines commercialisées :

- les opérations de micro-rectification sont effectuées sur une Nanoform 600 de la société Precitech (un tour à commande numérique d'ultra-précision), utilisée avec une tête de meulage également fournie par Precitech.

- La phase de finition magnéto-rhéologique est réalisée à l'aide d'une Q22 de la société QED. C'est la première machine à commande numérique de finition magnétorhéologique à avoir été commercialisée. Une description détaillée de cette technique est dans l'Annexe 2.

Quelle que soit la phase de fabrication, il est toujours nécessaire de programmer les déplacements relatifs de l'outil et de la pièce. Lors des phases de microrectification, la programmation est faite dans un premier temps à partir de la forme théorique du dioptre. Après quoi, le programme de déplacement est corrigé en fonction des défauts de forme constatés lors des mesures de contrôle bidimensionnelles. Dans le cas de la finition magnéto-rhéologique, le déplacement est piloté par le logiciel de la Q22 à partir d'une mesure de forme en trois dimensions du dioptre. La précision de la forme finale du dioptre est donc fonction de la précision de la mesure de la forme initiale de ce dioptre.

À chaque étape, des mesures précises sont indispensables à une fabrication de qualité et, à la fin, au contrôle du résultat obtenu. La précision finale du dioptre dépend donc étroitement des moyens de mesure associés aux moyens de production.

\subsubsection{Systèmes de mesures}

Durant les phases de micro-rectification, un profilomètre mécanique (Form Talysurf série 2 de Taylor Hobson) est utilisé et permet de faire des mesures de forme et de rugosité bidimensionnelles.

Lors des mesures de rugosité, une pointe en diamant en forme de cône est utilisée. Son rayon est de $2 \mu \mathrm{m}$ et le demi-angle au sommet du cône est de $30^{\circ}$. Le profilomètre ne peut évaluer une rugosité dont le paramètre $R a$ est inférieur à $10 \mathrm{~nm}$. La précision du paramètre de rugosité $R a$ mesuré par cet instrument est de $2 \%$, limitée à $\pm 4 \mathrm{~nm}$. Il est également possible d'utiliser le palpeur précédent pour réaliser des mesures de forme sur des dioptres dont la flèche est inférieure à $6 \mathrm{~mm}$, dans le cas contraire il faut utiliser un palpeur à bille dont le rayon de la bille est de $0,5 \mathrm{~mm}$. L'incertitude sur le paramètre de forme PRt est de $0,15 \mu \mathrm{m}$ avec le palpeur à pointe de diamant et de $0,25 \mu \mathrm{m}$ avec le palpeur à bille de saphir.

Les mesures de forme en trois dimensions sur les dioptres asphériques sont effectuées par un interféromètre de type Fizeau à décalage de phase (RTI 6100 de Wyko), dont la source lumineuse est un laser HeNe de longueur d'onde $633 \mathrm{~nm}$, avec un réseau à pas variable (CGH : Computer Genereted Hologram) qui est propre à un seul type d'asphérique. Les mesures données par l'interféromètre sont exactes à $\lambda / 200$ en $R t(3,2 \mathrm{~nm})$ et à $\lambda / 3000$ en rms $(0,2 \mathrm{~nm})$. Cependant, l'utilisation d'un réseau à pas variable $(\mathrm{CGH})$ fait que la précision des mesures du paramètre $P R t$ devient proche de $\lambda / 6(106 \mathrm{~nm})$.

Les mesures de rugosité en trois dimensions et sans contact sur les dioptres asphériques sont effectuées par un microscope interférométrique (Zygo New View 5020). Cet appareil utilise une lumière blanche, donc polychromatique. La précision sur les mesures de rugosité est, en $(x, y)$ fonction du grossissement de l'objectif utilisé (elle se situe entre 0,4 et $8 \mu \mathrm{m}$ ) et suivant $z$ inférieure à $1 \mathrm{~nm}$.

\subsubsection{Processus définitif de fabrication}

Une phase intermédiaire de polissage souple placée entre les phases de micro-rectification et celle de magnétorhéologie a été introduite, car des défauts de forme locaux créés par la deuxième phase de micro-rectification, ainsi qu'une rugosité supérieure aux exigences posées, subsistaient après la phase de finition magnéto-rhéologique (Fig. 1). L'objectif lors de cette phase intermédiaire est donc d'éliminer ces défauts de forme locaux et d'améliorer la rugosité, en utilisant un polissoir dont la surface active est souple; celle-ci peut donc se déformer pour suivre les variations d'un dioptre asphérique. Cependant, la déformation du dioptre entraînée par ce polissage ne doit pas être trop importante afin que la phase de finition magnéto-rhéologique ne soit pas trop longue. Il est donc admissible de tolérer, en fonction de la taille du dioptre, une déformation de 1 ou $2 \mu \mathrm{m}$ sur le paramètre $P R t$.

Parmi la dizaine de solutions techniques qui ont été envisagées pour effectuer cette phase, deux ont été retenues.

\subsubsection{Procédés de polissage souple sur des dioptres asphériques}

Les deux procédés décrits ci-dessous ont été mis au point dans le cadre de cette étude. 


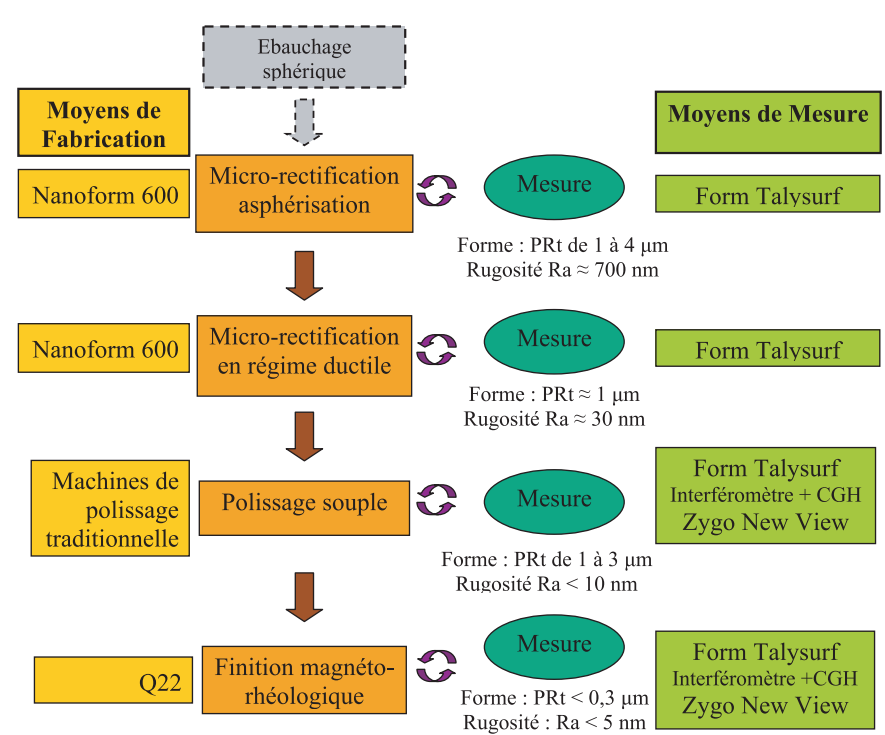

Fig. 1. Seconde approche du processus de réalisation d'un dioptre asphérique en verre

\subsubsection{Polissage tripode}

Ce polissage est réalisé sur une machine de polissage conventionnelle à l'aide de trois petits polissoirs, qui sont chacun reliés par une liaison rotule à une même pièce. Celle-ci maintient leur écartement et les met en mouvement. L'ensemble est appelé polissoir tripode (Fig. 2). Les petits polissoirs sont constitués d'un support rigide sur lequel sont collées une mousse en caoutchouc cellulaire étanche puis une feuille de polyuréthane (c'est cette feuille qui est en contact avec le dioptre à polir). La surface active des polissoirs a une forme sphérique au rayon de meilleure sphère de l'asphérique. Le tripode est posé sur la lentille qui est en rotation et exerce sur celle-ci un effort de $85 \mathrm{~N}$. Le polissoir est entraîné dans un mouvement de va-et-vient dans le plan horizontal de façon symétrique par rapport à l'axe de rotation de la lentille. Le liquide de polissage est composé d'abrasifs (oxydes de cérium et de terres rares) et d'eau; $90 \%$ des particules ont une taille inférieure au micromètre. L'arrosage est continu et se fait par le centre du tripode.

Du fait de la taille et de la conception d'un polissoir tripode, cette technique n'est applicable que pour les lentilles de grand diamètre (supérieur à $100 \mathrm{~mm}$ ).

\subsubsection{Polissage membrane}

Le polissage membrane consiste à utiliser comme surface active une membrane tendue (Fig. 3). Le polissoir est constitué d'un outil en laiton qui forme une cavité cylindrique fermée par une membrane composée d'une feuille de caoutchouc pour assurer son étanchéité et d'une feuille de polyuréthane. Par l'intermédiaire d'une valve, il est possible de créer une dépression ou une surpression dans la chambre, ce qui permet de donner à la surface active $\mathrm{du}$ polissoir une forme concave ou convexe.

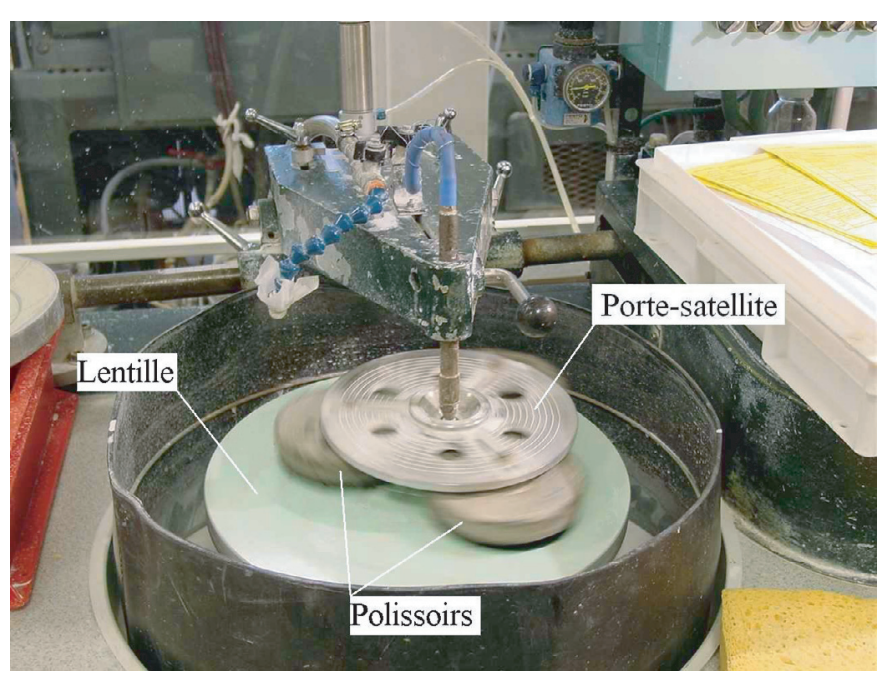

Fig. 2. Photo d'un polissoir tripode.

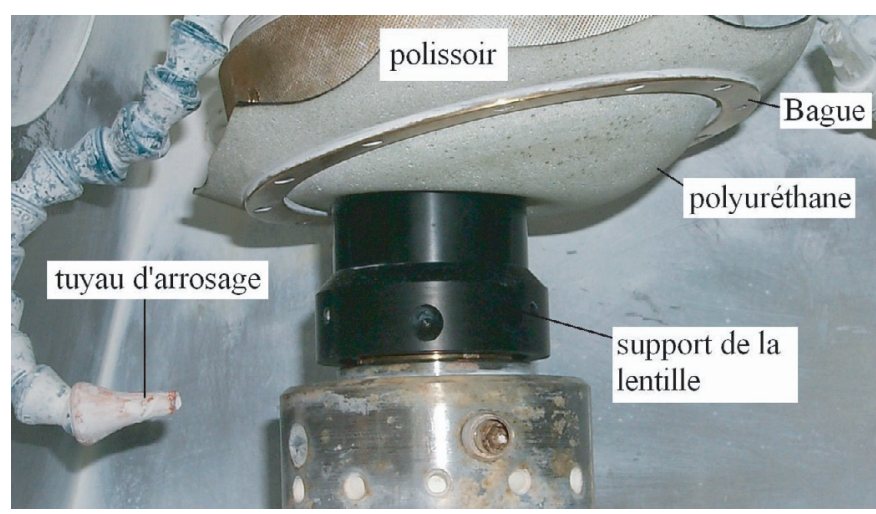

Fig. 3. Photo du polissoir membrane en position de polissage.

Le polissoir et la lentille sont en rotation autour de leur axe de symétrie. L'axe de rotation du polissoir est incliné de $2^{\circ}$ par rapport à l'axe de rotation de la lentille. Autour de cette position, le polissoir a un mouvement oscillatoire d'amplitude $6^{\circ}$. Le liquide de polissage est une solution aqueuse d'opaline (abrasif : oxyde de cérium; diamètre moyen des particules : $1 \mu \mathrm{m}$; $\mathrm{pH}$ de la solution : 6-7). Le polissoir membrane qui a été testé, ne permet pas de polir des dioptres dont le diamètre excède $50 \mathrm{~mm}$.

Avant de présenter les résultats obtenus en terme de rugosité et de forme, il est indispensable d'examiner le comportement du matériau pendant ces différentes étapes et de bien cerner les différents mécanismes d'abrasion du verre.

\section{Comportement des verres lors de la micro-rectification}

\subsection{Les matériaux utilisés dans cette étude}

Différents matériaux, transparents dans un intervalle de longueur d'onde compris entre $400 \mathrm{~nm}$ et $700 \mathrm{~nm}$, peuvent être utilisés pour la réalisation de lentilles 
possédant un dioptre asphérique dans le domaine du visible. On peut notamment citer les verres optiques, la fluorine et les polymères.

Les verres optiques sont les matériaux les plus employés chez Thales Angénieux. Au moins 335 verres y sont référencés, qui sont fabriqués par des verriers spécialisés (Ohara Optical Glass, Schott, Hoya, ...). Ils sont choisis par les ingénieurs opticiens qui conçoivent les systèmes optiques en fonction de leurs propriétés optiques (indice de réfraction, constringence). Les propriétés mécaniques (rigidité, dureté, abrasion) et les propriétés chimiques (réactivité de la surface, ...) sont également prises en compte. Certaines caractéristiques des différents verres qui ont été utilisés durant ce travail sont présentées dans l'Annexe 3.

\subsection{Les mécanismes d'enlèvement de la matière sur les verres optiques}

La réalisation de composants optiques peut faire appel à différents procédés de fabrication. Sur le verre les principaux procédés sont :

- la micro-rectification et le doucissage,

- le polissage et la finition magnéto-rhéologique.

Tous ces procédés mettent en jeu des mécanismes d'enlèvement de la matière qui sont des processus complexes impliquant des interactions mécaniques et chimiques entre la pièce, les abrasifs, le liquide d'arrosage et, s'il existe, le liant.

Nous nous intéresserons ici qu'aux mécanismes d'enlèvement de la matière en micro-rectification. Durant ces opérations d'enlèvement de la matière, deux régimes peuvent être mis en jeu en fonction de la taille des abrasifs, des efforts de coupe, du liquide de coupe et du type de matériau travaillé.

\subsubsection{Le régime cassant}

L'enlèvement de la matière en régime cassant permet d'ôter rapidement beaucoup de matière. Il est donc particulièrement intéressant d'un point de vue industriel pour mettre en forme des pièces. Cependant, il produit une surface qui est très « accidentée » et perturbée en profondeur. Les abrasifs, de par la pression qu'ils exercent sur le dioptre, créent un réseau de fissures sur toute la surface du substrat (Fig. 4). Ces fissures, en se rejoignant, produisent des éclats de verre.

Les valeurs du paramètre de rugosité $R t$ peuvent être de l'ordre de $10 \mu \mathrm{m}$. Sous la surface, la couche endommagée, engendrée par l'opération de fabrication peut avoir une épaisseur atteignant $100 \mu \mathrm{m}$.

Ce type de régime est donc caractérisé par [13-15] :

- une profondeur de coupe importante et des grains d'abrasif de grosse taille,

- un fort taux d'enlèvement matière,

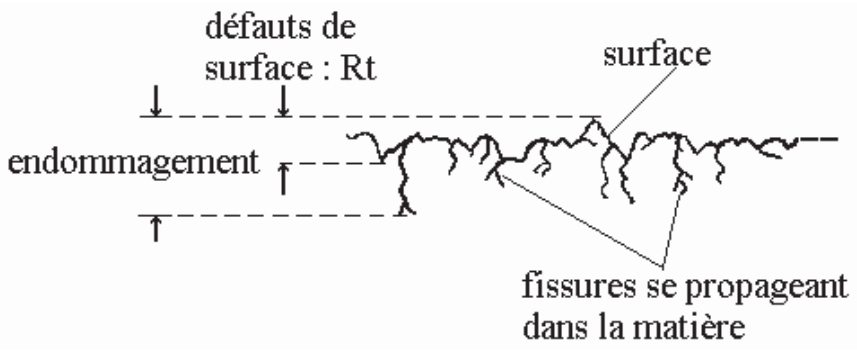

Fig. 4. Schéma de la structure d'une surface après une opération de micro-rectification en régime cassant.

- l'obtention d'une surface possédant une très forte rugosité qui augmente avec la taille des grains d'abrasif,

- un endommagement proportionnel au paramètre $R t$ de rugosité,

- de faibles contraintes résiduelles dans la couche supérieure de la pièce.

L'importance des défauts de surface et des endommagements dépend non seulement des propriétés du verre, mais également de tous les paramètres et conditions utilisés lors de la fabrication. Une telle surface est en général inutilisable, car elle diffuse la lumière. La couche endommagée doit donc être éliminée.

\subsubsection{Le régime ductile}

Les opérations de micro-rectification peuvent également être conduites en régime ductile (parfois appelé « régime de cisaillement »). Ce processus s'apparente à l'usinage des métaux [16]. Les forces exercées sur le substrat sont traduites par des forces latérales de cisaillement pratiquement parallèles à la surface. Le verre n'est plus ôté par l'intermédiaire de fissures, mais par une déformation plastique.

Les caractéristiques d'un régime ductile sont [13-16] :

- une faible profondeur de coupe ou des grains d'abrasif de petite taille,

- un taux d'enlèvement matière bien plus faible que lors d'opérations en régime cassant,

- l'obtention d'une surface possédant une faible rugosité,

- un très faible endommagement (quasiment nul), qui n'est plus proportionnel au paramètre de rugosité $R t$,

- de fortes contraintes résiduelles présentes dans la couche superficielle.

La micro-rectification en régime ductile permet de réduire fortement la profondeur de la couche endommagée et la rugosité de la surface. Ces améliorations entraînent une réduction considérable et une harmonisation du temps de polissage lors des phases suivantes. Cependant, une opération en régime ductile produit plus de contrainte dans la couche superficielle (effet de Twyman) [17] qu'en régime cassant. Cette différence s'explique par le fait qu'en régime cassant une grande partie de l'énergie est libérée lors de la fissuration du substrat, 


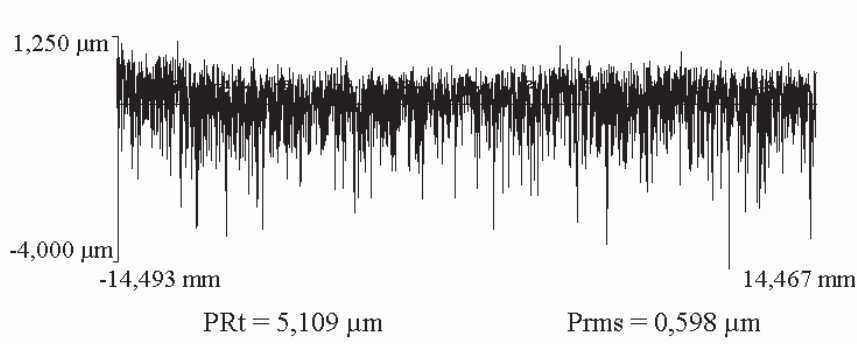

Fig. 5. Mesure de forme linéique d'un dioptre micro-rectifié en régime cassant (mesure Form Talysurf).

alors qu'en régime ductile, l'énergie est transformée en déformation permanente de la couche superficielle.

Il est également possible de rencontrer un troisième type de régime d'enlèvement de la matière, le régime ductile partiel, qui est un mélange du régime cassant et du régime ductile [18].

\section{Résultats expérimentaux et discussion}

\subsection{La première phase de micro-rectification}

L'objectif de cette phase est de former l'asphérique et d'éliminer la couche de matière endommagée par l'opération d'ébauchage. Avec la meule utilisée (liant métallique et gros grains de diamant : $30 \mu \mathrm{m})$, cette phase est réalisée sans difficulté par micro-rectification en régime cassant. Cependant un tel régime engendrant un très mauvais état de surface (Fig. 5) et un endommagement important, différents essais ont été réalisés avec ce type de meule pour obtenir un état de surface de bonne qualité qui permettrait de remplacer la seconde phase de micro-rectification par une passe de finition supplémentaire lors de cette première phase de microrectification. L'objectif était donc de déterminer des paramètres de coupe ou un environnement qui autoriserait une opération de micro-rectification en régime ductile avec une telle meule. Le passage du régime cassant au régime ductile peut se faire en diminuant l'énergie apportée par chaque grain de diamant afin que le processus de fissuration ne puisse pas avoir lieu, c'est-à-dire en modifiant les paramètres de coupe [19]. Mais il est également possible d'augmenter la résistance à la fissuration et les propriétés mécaniques et surfaciques de la pièce en modifiant l'environnement durant l'opération de micro-rectification, ce qui revient à augmenter l'énergie nécessaire à la fissuration du matériau [20]. Cependant aucun essai sur les paramètres de coupe n'a permis d'obtenir une micro-rectification en régime ductile sur tout le dioptre. Deux caractéristiques de la meule peuvent en partie expliquer cette difficulté :

- le liant métallique,

- les grains de grande dimension.

Bien que le liant métallique de cette meule soit l'un des plus doux qui puisse être utilisé, l'usure de ce liant reste

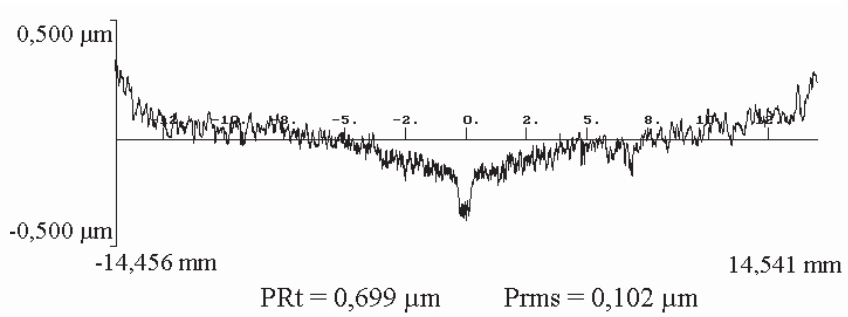

Fig. 6. Mesure de forme linéique d'un dioptre micro-rectifié en régime ductile (mesure Form Talysurf).

lente et donc le renouvellement des grains l'est aussi. Cela implique qu'une partie des grains actifs est émoussée, or de tels grains favorisent l'apparition de fissures [21].

Des essais ont également été réalisés avec des fluides de coupe composés soit d'eau pure, soit d'une solution aqueuse d'acide chlorhydrique de $\mathrm{pH} 4$, soit d'une solution aqueuse d'hydroxyde de sodium de $\mathrm{pH}$ 11. Dans les trois cas, les résultats ont été similaires à ceux obtenus avec le fluide habituel (constitué d'eau et de $4 \%$ d'huile hydrophile) en utilisant les mêmes paramètres de coupes. Ce résultat peut s'expliquer par le fait que les molécules d'eau sont très fortement majoritaires dans ces quatre liquides de coupe. Ce sont les caractéristiques de ces molécules (très petites et fortement polarisées) qui déterminent l'action de ces fluides lors des opérations de micro-rectification [22, 23].

Les résultats de ces essais étant peu concluants, le processus décrit à la section 2.3.4 a été adopté. La première phase de micro-rectification est donc effectuée en régime cassant. Des passes de $20 \mu \mathrm{m}$ de profondeur avec une vitesse d'avance de $20 \mathrm{~mm} . \mathrm{min}^{-1}$ sont réalisées.

\subsection{La seconde phase de micro-rectification}

Cette opération est utilisée pour améliorer l'état de surface et la forme du dioptre. Les objectifs de cette phase sont d'atteindre :

- une forme : PRt $\leq 1 \mu \mathrm{m}$,

- un état de surface : Ra de l'ordre de $20 \mathrm{~nm}$.

Pour arriver à une faible rugosité, cette phase de micro-rectification doit avoir lieu en régime ductile. Pour cela, la meule utilisée doit posséder des grains de diamant de petite dimension $(6 \mu \mathrm{m})$ et un liant résinoïde suffisamment tendre pour que les grains de diamant puissent être libérés de la meule avant qu'ils ne soient trop usés. Toutefois, un compromis sur la dureté du liant doit être trouvé pour que la meule ne se déforme pas trop vite. En utilisant les paramètres de coupes suivants : profondeur de passe $\leq 10 \mu \mathrm{m}$ et vitesse d'avance de $20 \mathrm{~mm} \cdot \mathrm{min}^{-1}$; les objectifs en forme et en rugosité de cette phase sont atteints (Fig. 6).

Cependant, de faibles défauts de forme subsistent sur le dioptre. Certes, ces défauts sont trop petits pour influencer la valeur des paramètres de forme et ils ne font 


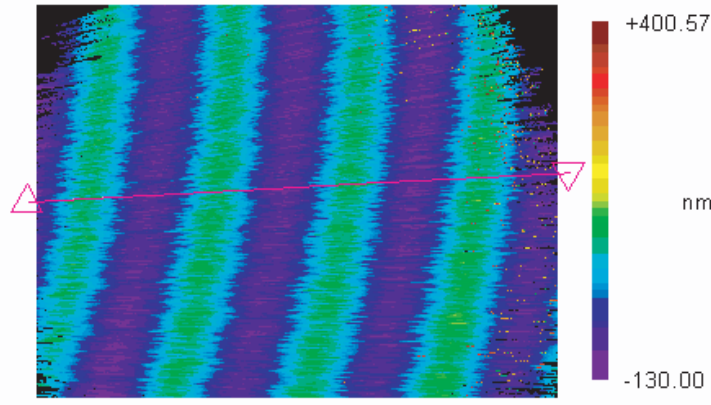

résultats de l'analyse surfacique : $\mathrm{Rt}=530,6 \mathrm{~nm}$ $\mathrm{rms}=58,84 \mathrm{~nm}$ $\mathrm{Ra}=50,62 \mathrm{~nm}$

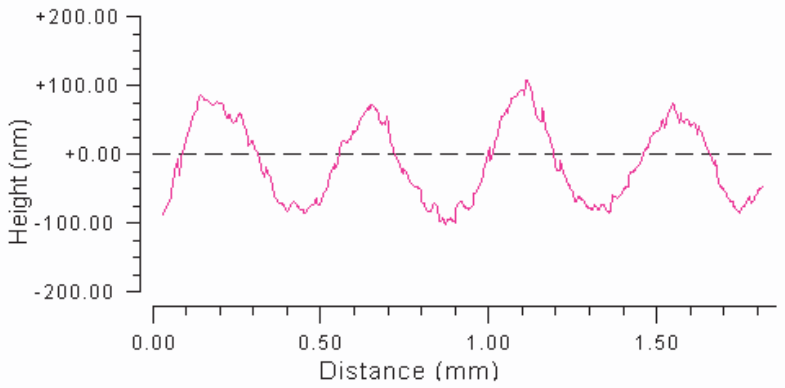

résultats de l'analyse linéique $:$ Rt $=209,6 \mathrm{~nm}$

$\mathrm{rms}=57,14 \mathrm{~nm}$

$\mathrm{Ra}=51,14 \mathrm{~nm}$

Fig. 7. Mesure de rugosité des défauts d'ondulation sur un dioptre micro-rectifié en régime ductile (surface de mesure $0,58 \mathrm{~mm}^{2}$, rugosimètre optique Zygo).

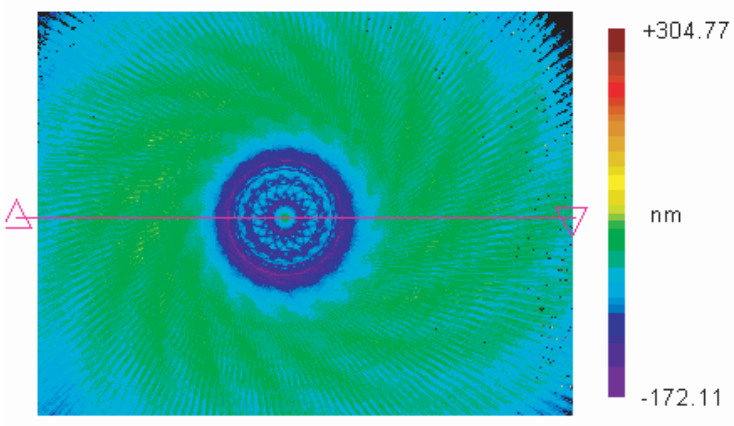

résultats de l'analyse surfacique : $\mathrm{Rt}=476,9 \mathrm{~nm}$ $\mathrm{rms}=29,84 \mathrm{~nm}$ $\mathrm{Ra}=21,28 \mathrm{~nm}$

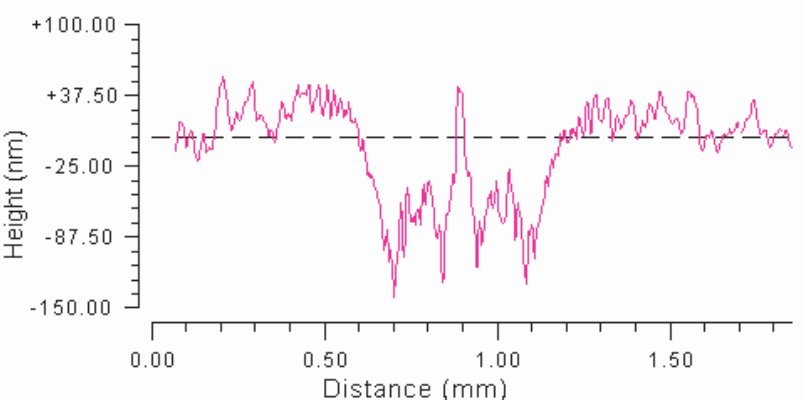

résultats de l'analyse linéique

$$
\begin{aligned}
& \mathrm{Rt}=194,0 \mathrm{~nm} \\
& \mathrm{rms}=42,08 \mathrm{~nm} \\
& \mathrm{Ra}=34,38 \mathrm{~nm}
\end{aligned}
$$

Fig. 8. Mesure de rugosité au centre d'un dioptre micro-rectifié en régime ductile (surface de mesure $0,58 \mathrm{~mm}^{2}$, rugosimètre optique Zygo).

pas augmenter les paramètres de rugosité suffisamment pour remettre en cause le passage à la finition magnétorhéologique. Néanmoins, ces défauts ne sont pas éliminés par cette dernière phase et ne sont pas acceptables pour des produits de Thales Angénieux. Ces défauts, appelés défauts locaux de forme, sont de deux types :

- La majorité d'entre eux sont apparentés à des défauts d'ondulation. Leur amplitude peut aller jusqu'à 200 nm (Fig. 7). Différents essais sur les conditions de coupe (vitesse d'avance, vitesse de rotation de la meule et de la pièce, profondeur de coupe) nous ont permis de réduire ces défauts. Ces essais ont d'ailleurs montré la grande importance de l'équilibrage dynamique de la meule. Lors de la mise en place d'une meule à liant résinoïde, la meule est dressée, puis la partie en rotation de la tête de meulage est équilibrée. Lorsque l'équilibrage dynamique est optimisé, cela limite l'amplitude des défauts d'ondulation à un niveau d'environ $50 \mathrm{~nm}$.

- Un autre défaut subsiste également au centre des dioptres [24]. Il a la forme d'un trou circulaire dont le diamètre est de l'ordre de $100 \mu \mathrm{m}$ (Fig. 8), sa profondeur est en général comprise entre 0,1 et $0,2 \mu \mathrm{m}$. Ce défaut n'est pas éliminé par les moyens de correction habituels utilisés lors des phases de micro-rectification (compensation sur le programme de déplacement de la meule). Une des hypothèses les plus probables pour expliquer ce phénomène est que durant toute cette phase, la meule est comprimée par la pièce. Lorsqu'elle s'éloigne de celle-ci, la contrainte se relâche, mais le contact entre la pièce et la meule est prolongé par le fait que la meule retrouve progressivement sa taille d'origine.

À l'issue de la phase de micro-rectification en régime ductile et malgré les ajustements réalisés sur les paramètres de coupe, les défauts locaux de forme sont toujours présents et nous ont obligés à ajouter une phase de polissage souple. Suite à l'introduction de cette nouvelle phase, les objectifs que doit atteindre la seconde phase de micro-rectification sont dorénavant :

- Paramètre de forme : PRt $\leq 1 \mu \mathrm{m} \pm 0,5 \mu \mathrm{m}$,

- Paramètre de rugosité : $R a$ compris entre 20 et $50 \mathrm{~nm}$. 

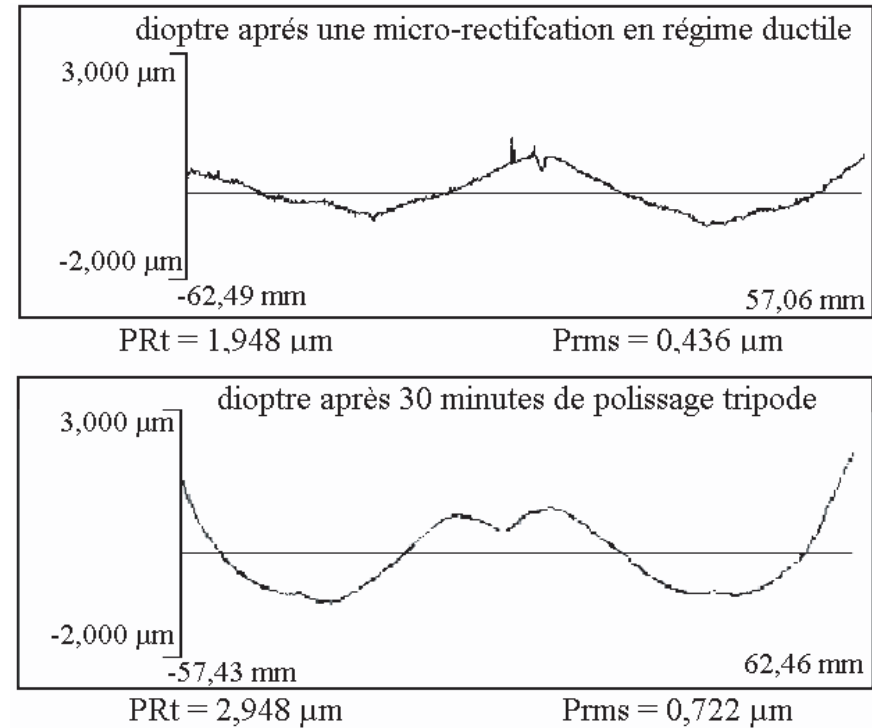

Fig. 9. Mesure de forme d'un dioptre micro-rectifié en régime ductile avant et après le polissage tripode (mesure Form Talysurf).

\subsection{Phases intermédiaires de polissage souple}

\subsubsection{Polissage tripode}

Les essais de polissage tripode ont été réalisés sur des dioptres de grand diamètre $(200 \mathrm{~mm})$ et de forte asphéricité (1,35 $\mathrm{mm}$ ) micro-rectifiés en régime ductile.

L'élimination des défauts de forme locaux (ondulation et trou central) a nécessité $30 \mathrm{~min}$ de polissage tripode. À la suite de ce polissage, la rugosité des dioptres est correct : le paramètre $R a$ de rugosité a été amené à une valeur moyenne de $2 \mathrm{~nm}$. En revanche, ce procédé a dégradé la forme des dioptres. Le paramètre de forme PRt est passé en moyenne d'une valeur de $2 \mu \mathrm{m}$ à $3 \mu \mathrm{m}$ et le Prms de $0,4 \mu \mathrm{m}$ à $0,8 \mu \mathrm{m}$ (Fig. 9).

\subsubsection{Polissage membrane}

Les essais avec ce procédé de polissage souple ont été faits sur des dioptres d'asphéricité $80 \mu \mathrm{m}$ et de diamètre $32 \mathrm{~mm}$.

Ces essais ont mis en évidence qu'une importante phase de réglage des paramètres de polissage est nécessaire au début du polissage de chaque nouveau type de dioptre asphérique. Les principaux paramètres sont le rayon de courbure du polissoir (la pression dans le polissoir), l'enfoncement du dioptre dans le polissoir et l'angle d'oscillation du polissoir autour l'axe du dioptre. Le polissage membrane permet d'éliminer les défauts de forme locaux et d'améliorer la rugosité. Un polissage de cinq minutes suffit dans la majorité des cas pour obtenir un état de surface autorisant le passage à la phase de finition magnéto-rhéologique. Le paramètre de rugosité est alors, de l'ordre de $3 \mathrm{~nm}$. En contrepartie, la déformation du dioptre est au maximum d'environ $1 \mu \mathrm{m}$ sur le paramètre PRt (Fig. 10).
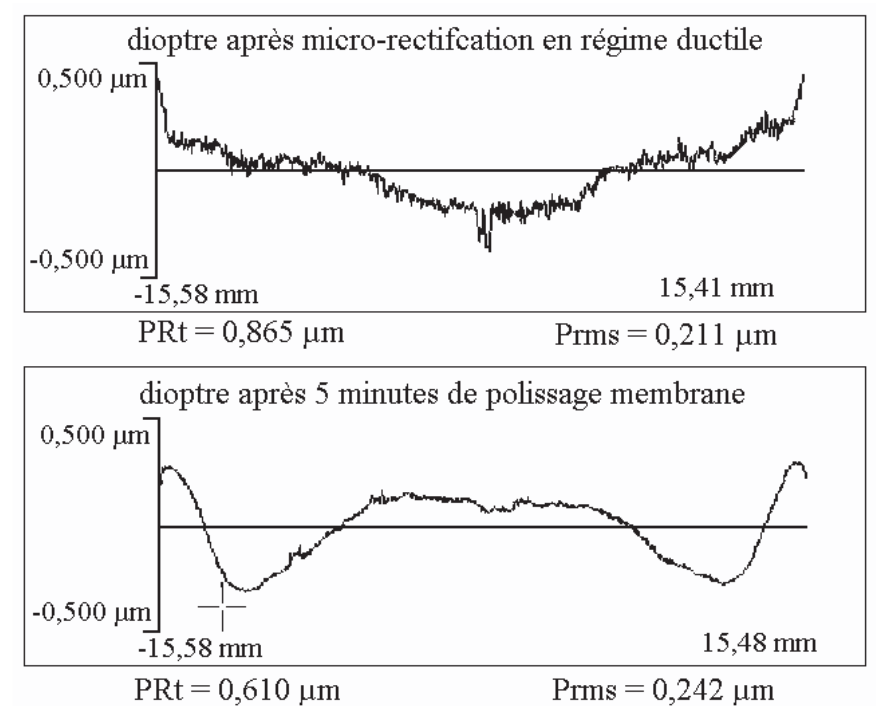

Fig. 10. Mesure de forme d'un dioptre micro-rectifié en régime ductile et mesure de forme du même dioptre après 5 min de polissage membrane (mesure Form Talysurf).

\subsubsection{Conclusion sur cette phase}

Les deux techniques de polissage souples présentées répondent aux exigences de cette phase : élimination des défauts de forme locaux, amélioration de la rugosité $(R a<5 \mathrm{~nm})$ et déformation du dioptre inférieure à $1 \mathrm{ou}$ $2 \mu \mathrm{m}$ sur le paramètre de forme PRt. Ces deux techniques sont complémentaires :

- le polissage tripode est destiné aux dioptres asphériques de grand diamètre (supérieur à 100 mm),

- le polissage membrane aux dioptres asphériques de petit diamètre (inférieur à $100 \mathrm{~mm}$ ).

Cette distinction, entre petits et grands diamètres, est imposée par les technologies employées dans chacun de ces deux procédés. Cependant, la limite de $100 \mathrm{~mm}$ est approximative et pourrait être modifiée.

Toutes les méthodes de polissage souple testées déforment le dioptre. Afin de remédier à cet inconvénient, il est possible pour les séries de dioptres relativement importantes d'évaluer le défaut de forme généré par la méthode de polissage souple employée et d'introduire l'opposé de ce défaut dans la trajectoire de la meule lors de la phase de micro-rectification en régime ductile. Cela permettrait alors de réduire le temps de cycle de la phase de finition.

\subsection{Finition magnéto-rhéologique}

Les essais de finition magnéto-rhéologique sur des dioptres asphériques de petits diamètres ayant subi une phase de polissage souple donnent d'excellents résultats (Fig. 11). Avec une fonction d'enlèvement matière adéquate, les temps de cycle varient de $10 \mathrm{~min}$ à $25 \mathrm{~min}$ en fonction du défaut à corriger (défaut dont le PRt est 


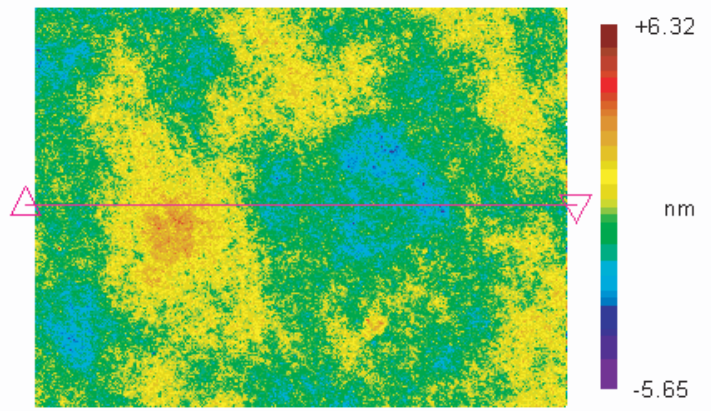

résultats de l'analyse surfacique : Rt $=12 \mathrm{~nm}$

$\mathrm{rms}=0,92 \mathrm{~nm}$

$\mathrm{Ra}=0,73 \mathrm{~nm}$

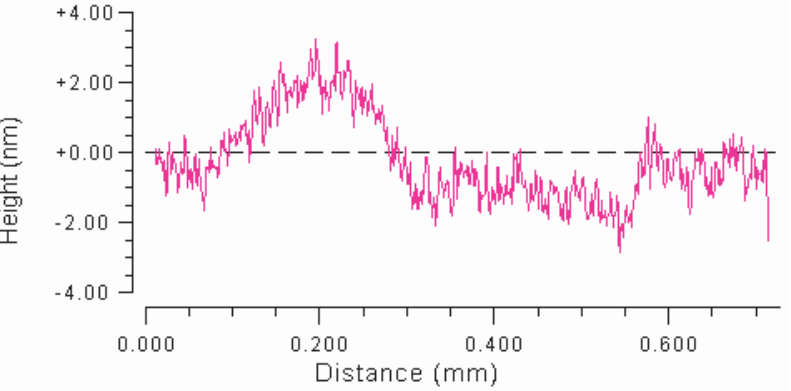

résultats de l'analyse linéique $: \mathrm{Rt}=6,1 \mathrm{~nm}$

$\mathrm{rms}=1,21 \mathrm{~nm}$

$\mathrm{Ra}=0,97 \mathrm{~nm}$

Fig. 11. Dioptre asphérique poli souple puis fini sur la Q22 (surface de mesure $0,37 \mathrm{~mm}^{2}$, rugosimètre optique Zygo).

Tableau 1. Les différentes étapes du processus ainsi que l'état du dioptre à la fin de chaque phase.

\begin{tabular}{|c|c|c|c|c|}
\hline Opération & Forme & Rugosité & $\begin{array}{l}\text { Aspect du dioptre à } \\
\text { la fin de l'opération }\end{array}$ & $\begin{array}{l}\text { Machine } \\
\text { utilisée }\end{array}$ \\
\hline Ébauchage & $\begin{array}{l}P R t \approx 10 \mu \mathrm{m} \\
P R m s \approx 1,5 \mu \mathrm{m}\end{array}$ & $R a \approx 1000 \mathrm{~nm}$ & Dioptre sphérique & $\begin{array}{l}\text { Machine de } \\
\text { doucissage } \\
\text { traditionnelle }\end{array}$ \\
\hline $\begin{array}{l}1^{\circ} \text { phase de micro- } \\
\text { rectification }\end{array}$ & $\begin{array}{l}P R t \approx 4 \mu \mathrm{m} \\
P R m s \approx 0,6 \mu \mathrm{m}\end{array}$ & $R a \approx 700 \mathrm{~nm}$ & $\begin{array}{l}\text { Dioptre asphérique } \\
\text { translucide }\end{array}$ & Nanoform 600 \\
\hline $\begin{array}{l}2^{\circ} \text { phase de micro- } \\
\text { rectification }\end{array}$ & $\begin{array}{l}P R t \approx 1 \mu \mathrm{m} \\
P R m s \approx 0,2 \mu \mathrm{m}\end{array}$ & $R a \approx 50 \mathrm{~nm}$ & $\begin{array}{l}\text { Dioptre asphérique } \\
\text { transparent avec } \\
\text { défauts locaux } \\
\text { de forme }\end{array}$ & Nanoform 600 \\
\hline Polissage souple & $\begin{array}{l}P R t \approx 2 \mu \mathrm{m} \\
P R m s \approx 0,4 \mu \mathrm{m}\end{array}$ & $R a<10 \mathrm{~nm}$ & $\begin{array}{l}\text { Dioptre asphérique } \\
\text { transparent sans } \\
\text { défauts locaux } \\
\text { de forme }\end{array}$ & $\begin{array}{l}\text { Machine de } \\
\text { polissage } \\
\text { sphérique }\end{array}$ \\
\hline $\begin{array}{l}\text { Finition magnéto- } \\
\text { rhéologique }\end{array}$ & $\begin{array}{l}P R t \approx 0,2 \mu \mathrm{m} \\
P R m s<0,03 \mu \mathrm{m}\end{array}$ & $R a<5 \mathrm{~nm}$ & Dioptre asphérique & Q22 \\
\hline
\end{tabular}

inférieur ou égal à $1 \mu \mathrm{m})$. L'épaisseur minimum enlevée est comprise entre $0,12 \mu \mathrm{m}$ et $0,25 \mu \mathrm{m}$. À l'issue de ces essais, les paramètres de forme et de rugosité des dioptres sont :

- Paramètres de forme :

- PRt de l'ordre de 0,200 $\mu \mathrm{m}$ pour des valeurs initiales comprises entre $0,379 \mu \mathrm{m}$ à $1,020 \mu \mathrm{m}$,

- Prms de 0,020 à 0,026 $\mu \mathrm{m}$ pour des valeurs initiales allant de $0,070 \mu \mathrm{m}$ à $0,350 \mu \mathrm{m}$,

- Paramètre de rugosité :

- Ra inférieur à $3 \mathrm{~nm}$.

Le tableau 1 récapitule les différentes étapes du processus ainsi que l'état du dioptre à la fin de chaque phase.

Cette phase de finition magnéto-rhéologique n'a pas été réalisée sur des dioptres asphériques de grand diamètre, car nous ne disposions pas d'un réseau à pas variable $(\mathrm{CGH})$ adapté à un dioptre de ce type. Néanmoins des essais ont été réalisés sur des dioptres sphériques de grand diamètre, essais qui ont donné des résultats tout à fait concluants.

\section{Conclusion}

La production de dioptres asphériques en verre ne peut utiliser les techniques de polissage sphérique conventionnelles qui font toutes appel aux propriétés géométriques de la sphère pour le déplacement du polissoir. C'est pourquoi il a été nécessaire de développer un nouveau processus de fabrication adapté à ces dioptres.

L'introduction des machines à commande numérique de très grande précision dans le domaine de la fabrication optique ainsi que le développement puis la commercialisation d'une machine de finition magnéto-rhéologique furent les deux événements autorisant le développement de ce nouveau processus de fabrication industrielle de dioptres asphériques en verre, basé sur une phase de microrectification et une phase de finition magnéto-rhéologique. Cependant, il a fallu optimiser chaque phase et assurer la transition de l'une à l'autre. Ce dernier point a demandé l'introduction d'une phase de polissage souple avant la finition magnéto-rhéologique. Cette phase intermédiaire permet d'éliminer les défauts locaux de forme générés par la micro-rectification en régime ductile et d'améliorer la 
rugosité du dioptre. Ainsi ce processus permet de produire de façon industrielle des dioptres asphériques.

Ce processus de fabrication peut encore être amélioré. Deux directions de développement doivent être privilégiées :

- D'une part, il semble possible de réduire les deux phases de micro-rectification en une seule et/ou d'éliminer la phase de polissage souple :

- Soit en maîtrisant mieux le processus de microrectification. Pour cela, des essais complémentaires doivent être faits sur l'influence de l'environnement sur le mode d'enlèvement de la matière (utilisation de fluide formant avec le matériau usiné un couple dont le potentiel zêta est nul [22]). Le procédé de micro-rectification ELID (ELectrolytic In-process Dressing [25]) est une autre voie de développement dans ce même sens.

- Soit en travaillant sur la rigidité de la tête de meulage et l'équilibrage de la meule.

- D'autre part, la mesure surfacique des asphériques lors de la phase de finition magnéto-rhéologique peut être aussi améliorée. En effet, seule la précision des mesures en trois dimensions des surfaces asphériques limite la précision de la forme asphérique obtenue à la fin de cette phase. Actuellement, différents travaux sont menés pour améliorer ce type de mesure (en particulier des travaux sur la mesure interférométrique de surfaces asphériques sans l'utilisation d'un réseau à pas variable, ...).

\section{Annexe 1 : Définition d'une surface asphérique}

Les surfaces asphériques abordées dans cet article sont des surfaces de révolution. Elles sont définies par une de leurs méridiennes dont l'équation est de la forme :

$$
z=\frac{y^{2}}{R\left(1+\sqrt{1-(1+k)\left(\frac{y^{2}}{R^{2}}\right)}\right)}+\sum_{i=2}^{n} A_{2 i} y^{2 i}
$$

- $R$ est le rayon osculateur de l'asphérique. Il correspond au rayon de courbure au niveau de l'axe de rotation de la surface;

- $A_{2 i}$ sont des constantes réelles;

- $k$ est le coefficient de conicité.

\section{Remarques :}

- Nous utilisons couramment la notion de meilleure sphère lorsque nous travaillons sur des surfaces asphériques (Fig. 12). La meilleure sphère d'un asphérique est telle qu'elle est tangente au centre de l'asphérique et sécante (en A) sur le diamètre utile de la lentille. Le rayon de la meilleure sphère est en général différent de $R$.

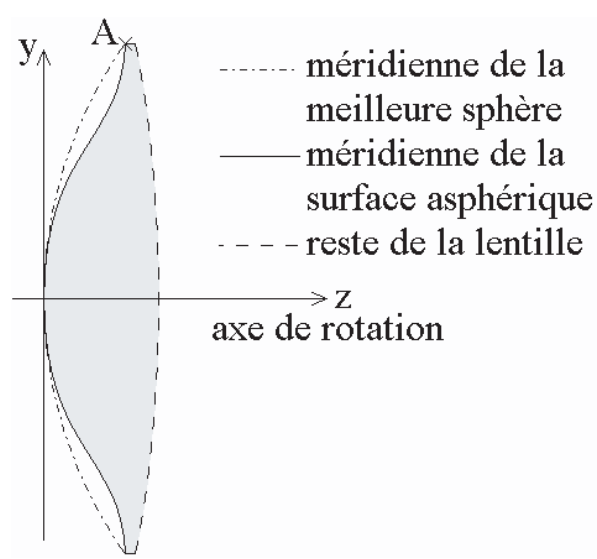

Fig. 12. Exemple d'une lentille ayant un dioptre asphérique.

- Nous employons également le terme d'asphéricité. L'asphéricité est l'écart maximum entre la surface asphérique et sa meilleure sphère parallèlement à l'axe $z$.

Le dioptre asphérique représenté sur la figure 12 est convexe. Un dioptre asphérique peut être concave.

\section{Annexe 2 : Principe de la finition magnéto-rhéologique}

Le polissage à l'aide d'un vecteur magnétique est né de la volonté, d'une part, de réduire le coût des outillages nécessaires à un atelier d'optique et d'autre part, de polir des dioptres non-sphériques avec la même précision que les dioptres sphériques. En effet, un polissoir, un rodoir et une contre-forme sont nécessaires pour polir chaque type de dioptre (en fonction du rayon et de sa concavité ou de sa convexité). L'idée était d'inventer un polissoir qui puisse diminuer le nombre des outils. Ce polissoir devait être petit et avoir une forme qui pourrait être modifiée en fonction des besoins. Ces deux caractéristiques permettent de pouvoir polir un grand nombre de dioptres différents sphériques et asphériques. L'utilisation d'un fluide magnétique pour véhiculer des particules abrasives et donc polir fut une des voies de recherche pour créer ce polissoir [12].

Ce type de polissage est appelé MRF pour « magnetorheological finishing ». Le principe de la finition magnétorhéologique est de mettre en contact le dioptre avec un fluide en mouvement ayant des propriétés magnétique et rhéologique. Au niveau de la zone de contact, la viscosité du fluide est fortement augmentée par un champ magnétique ce qui permet au fluide d'avoir une action d'enlèvement de matière significative. De plus, l'action de l'outil de polissage (c'est-à-dire du fluide) est essentiellement tangentielle au dioptre contrairement à celle d'un polissoir traditionnel qui exerce un effort normal à la surface. Dans le cas du polissage conventionnel, au-delà d'une certaine intensité des efforts normaux, les particules abrasives peuvent fissurer le substrat. Or, le seuil d'intensité avant la fissuration pour des efforts tangentiels est 
bien plus élevé que dans le cas d'efforts normaux. Ainsi, le polissage magnéto-rhéologique présente l'avantage de réduire considérablement les risques d'endommagement de la couche supérieure du dioptre $[10,11]$.

La machine Q22 de la société QED est la première version d'une machine de finition de dioptre optique de très grande précision utilisant un fluide magnéto-rhéologique. La Q22 est une machine-outil à commande numérique à quatre axes (deux axes de translation et deux axes de rotation). Ces axes permettent de déplacer la zone de contact entre le dioptre et le fluide sur l'ensemble de la surface à polir. Les déplacements axiaux ont une résolution de l'ordre de $1 \mu \mathrm{m}$; les rotations se font avec une résolution de $4 \mathrm{~s}$ d'arc.

Le fluide magnéto-rhéologique est composé essentiellement d'eau, de particules abrasives et de particules magnétiques. Les abrasifs peuvent être de l'oxyde de cérium ou des particules de diamant.

Le fluide est en circulation permanente dans la machine. S'il n'est plus en mouvement, il sédimente et devient impropre au polissage, car il risque très probablement de marquer le dioptre (frayure, filandres) et de boucher le circuit où il circule.

Le fluide est mis en mouvement par un système de distribution qui mesure en temps réel deux paramètres : sa pression et son débit. Dans la zone de travail, le fluide passe sur une roue en rotation qui l'entraîne. À ce niveau, un électro-aimant crée un champ magnétique d'à peu près $0,1 \mathrm{~T}$ qui va augmenter la viscosité du fluide et donc le durcir. En même temps, ce champ magnétique donne au fluide la forme d'un cordon (d'environ $2 \mathrm{~mm}$ de hauteur sur $6 \mathrm{~mm}$ de largeur) sur la roue (Fig. 13).

Le dioptre à polir est déplacé par rapport au sommet de la roue de telle sorte que les enlèvements de matière permettent d'atteindre la forme souhaitée. La trajectoire

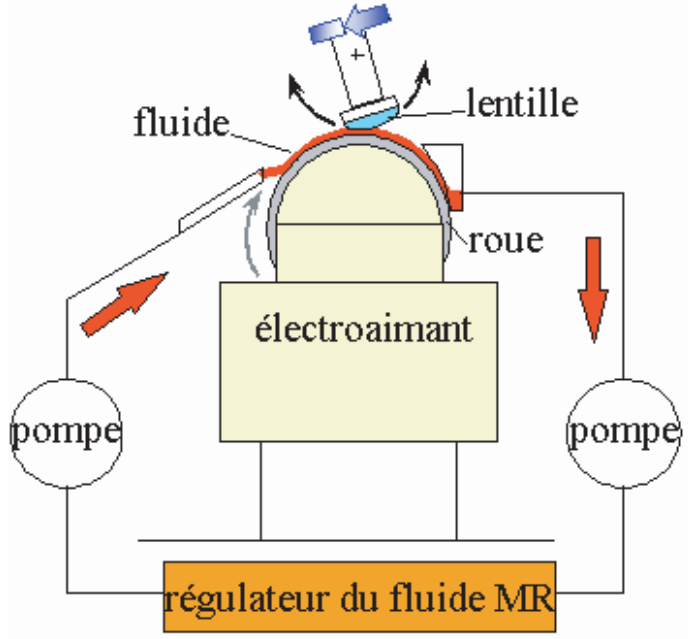

Fig. 13. Principe de fonctionnement de la Q22.

du dioptre est calculée par le logiciel de la Q22 en fonction de trois paramètres :

- la fonction d'enlèvement matière par le fluide sur le matériau composant le dioptre,

- la forme initiale du dioptre,

- la forme finale à atteindre.

Plusieurs cycles de finition magnéto-rhéologique peuvent être effectués sur un dioptre. Ces itérations permettent d'affiner les paramètres de déplacement du dioptre afin d'obtenir une très grande qualité de forme (défaut de forme inférieur à $30 \mathrm{~nm}$ en PRt).

Tableau 2. Les principaux verres étudiés et quelques-unes de leurs propriétés.

\begin{tabular}{|c|c|c|c|c|c|c|c|}
\hline matière & $\begin{array}{c}\text { Indice de } \\
\text { réfraction } \\
n_{\mathrm{d}}\end{array}$ & $\begin{array}{l}\text { Nombre } \\
\text { d'Abbe }\end{array}$ & $\begin{array}{c}\text { Module } \\
\text { d'élasticité } \\
\text { en MPa }\end{array}$ & $\begin{array}{c}\text { Micro- } \\
\text { dureté en } \\
\text { kg.mm }{ }^{-2}\end{array}$ & Abrasion & Fabricant & Lentille \\
\hline SK 2 & 1,60738 & 56,65 & 78000 & 460 & $\approx 130$ & Schott & $\begin{array}{c}\text { Test } \\
\text { asphérique }\end{array}$ \\
\hline SK 16 & 1,62041 & 60,33 & 89000 & 490 & 130 & Schott & $\begin{array}{c}\text { Test } \\
\text { asphérique }\end{array}$ \\
\hline PBH 6 & 1,805177 & 25,4 & 54300 & 340 & 212 & Ohara & $\begin{array}{l}\text { Ménisque } \\
\text { asphérique }\end{array}$ \\
\hline S-LAH 60 & 1,83400 & 37,2 & 124800 & 670 & 78 & Ohara & $\begin{array}{l}\text { Ménisque } \\
\text { asphérique }\end{array}$ \\
\hline S-FLP 51 & 1,49700 & 81,6 & 72900 & 350 & 439 & Ohara & $\begin{array}{c}\text { Test } \\
\text { sphérique }\end{array}$ \\
\hline S-FLP 53 & 1,43875 & 95,0 & 69400 & 360 & 389 & Ohara & $\begin{array}{c}\text { Test } \\
\text { sphérique }\end{array}$ \\
\hline S-BAL 35 & 1,58913 & 61,2 & 83200 & 590 & 115 & Ohara & $\begin{array}{c}\text { Test } \\
\text { sphérique }\end{array}$ \\
\hline S-BSL 7 (BK 7) & 1,51633 & 64,1 & 80000 & 570 & 94 & Ohara & $\begin{array}{c}\text { Test } \\
\text { sphérique }\end{array}$ \\
\hline Herasil I (silice) & 1,45840 & 67,8 & 72500 & 590 & & & Test plan \\
\hline
\end{tabular}




\section{Annexe 3 : Verres utilisés}

De façon générale, le constituant principal des verres optiques est la silice (60-70\%) qui est un matériau amorphe auquel les verriers ajoutent des éléments qui modifient les propriétés optiques du verre, mais aussi ses caractéristiques physiques (température de transition vitreuse) : oxydes de calcium, de sodium, de potassium, de baryum, de magnésium. Il peut y avoir également des traces d'arsenic et d'antimoine. Les verres étudiés et quelques unes de leurs propriétés sont présentés dans le tableau 2 [26, 27].

\section{Références}

[1] W. König, M. Weck, N. Spenrath, J. Luderich, Tutorial on diamond machining technology, 6th IPES/UME 2, Braunschweig, 1991

[2] F. Vega, J. Armengol, N. Lupón, F. Laguaria, Surface Dynamics during laser polishing of glass, SPIE, 3822, Munich, Germany, 1999, pp. 92-102

[3] O. Graydon, Abrasive jets shape and polish optics, Opto \& Laser Europe (1999) 18-31

[4] B.G. Bovard, Tianji Zhao, H. Angus Macleod, Oxygenion beam polishing of a 5-cm-diamond film, Applied Optics 31 (1992) 2366-2247

[5] J. P. Marioge, Surface asphérique, édition EDP Sciences, 2000

[6] G.-H. Chen, D.T. Moore, Asphérical surface polishing with a ring polisher, Applied Optics 18 (1979) 559-562

[7] J. Angénieux, A. Masson, Y. Rouchouse, Aspherics : precision manufacturing by vacuum evaporation, Optical Engineering 24 (1985) 499-501

[8] Document technique fourni par Toshiba : Toshiba GMP Series: Glass Molding Press, Toshiba machine Co., Ltd.

[9] Y. Namba, M. Ane, Ultraprecision grinding of optical glasses to produced supersmooth surfaces, Ann. CIRP 42 (1993) 417-420

[10] S.D. Jacob, D. Golini, Yuling Hsu, B.E. Puchebner, D. Strafford, I.V. Prokhorov, E.M. Fess, D. Pietrowsk, W.I. Kordonski, Magnetorheological finishing : a deterministic process for optics manufacturing, proceedings SPIE 2576 (1995) 372-382

[11] A.B. Shorey, S.D. Jacobs, W.I. Kordonski, R.F. Gans, Experiments and Observations Regarding the Mechanisms of Glass Removal in Magnetorheological Finishing, Applied Optics 40 (2001) 20-33
[12] Y. Saito, H. Niikura, T. Oshio, T. Hanaoka, Float polishing using magnetic fluid with abrasive grains, Proceedings of 6 th international conference production engineering, Osaka, 1987

[13] N.J. Brown, The response of isotropic brittle materials to abrasive processes, $43^{\circ}$ annual Symposium on Frequency Control, IEEE, New York, 1989, pp. 611-616

[14] D.F. Edwards, P.P. Hed, Optical glass fabrication technology (2): Relationship between surface roughness and subsurface damage, Applied Optics 26 (1987) 4677-4680

[15] O. Podzimek, Residual stress and deformation energy under ground surfaces of brittle solids, Ann. CIRP 35 (1986) 397-400

[16] D. Golini, S. D. Jacobs, Physics of loose abrasive microgrinding, Applied Optics 30 (1991) 2761-2777

[17] J.C. Lambropoulos, Su Xu, Tong Fang, D. Golini, Twyman effect mechanics in grinding and microgrinding, Applied Optics 35 (1996) 5704-5713

[18] Zhaowei Zhong, Partial-ductile grinding, lapping, and polishing of aspherical surfaces on glass, Materials and Manufacturing Process 12 (1997) 1063-1073

[19] M. Buijs, K. Korpel-Van Houten, A model for lapping of glass, J. Mat. Sci. 28 (1993) 3014-3020

[20] J.C. Lambropoulos, S.D. Jacobs, J. Ruckman, Material removal mechanics from grinding to polishing, Proceeding of Finishing of advanced ceramics and glasses, Indianapolis, Indiana, 1999, pp. 113-126

[21] S.S. Chiang, D. B. Marshall, A.G. Evans, The response of the solids to elastic/plastic indentation, J. Appl. Phys. 53 (1982) 298-311

[22] T.G. Bifano, D.K. Depiero, D. Golini, Chemomechanical effects in ductile-regime machining of glass, Precision engineering 15 (1993) 238-247

[23] J.C. Lambropoulos, Tong Fang, P.D. Funkenbusch, A.D. Jacobs, M.J. Cumbo, D. Golini, Surface microroughness of optical glasses under deterministic microgrinding, Applied Optics 35 (1996) 4448-4462

[24] D. Rollins, S.M. Gracewski, P. Funkenbusch, Process Parameter Optimization during Contour Grinding, Convergence 10 (2002) 1-3

[25] H. Ohmori, « Electrolytic In-Process Dressing (ELID) Grinding for Optical Parts Manufacturign », Int. Progress in Precision Eng., IPES7 (1993) 131-134

[26] Catalogue des verres optiques de la société Ohara Optical Glass, Inc.

[27] Catalogue des verres optiques de la société Schott, Schott Glass Technologies Inc. 